\title{
Flexibility and Technology Choice in Gas Fired Power Plant Investments
}

\author{
Erkka Näsäkkälä ${ }^{1}$, Stein-Erik Fleten ${ }^{2}$
}

\begin{abstract}
The value of a gas fired power plant depends on the spark spread, defined as the difference between the unit price of electricity and the cost of gas. We model the spark spread using two-factor model, allowing mean-reversion in short-term variations and uncertainty in the equilibrium price to which prices revert. We analyze two types of gas plants. The first type is a base load plant, generating electricity at all levels of spark spread. The second type is also a base load plant from the outset, but can be upgraded, at a cost, to a peak load plant generating electricity only when spark spread exceeds emission costs. We compute optimal building and upgrading thresholds for such plants when the plant types are mutually exclusive. Our results indicate that selecting a project which is first profitable leads to a nonoptimal investment policy, and that increase in short-term volatility preempts upgrading whereas increase in equilibrium volatility delays upgrading.
\end{abstract}

Key words: Real options, spark spread, gas fired power plants, investment flexibility, mutually exclusive projects

\footnotetext{
${ }^{1}$ Corresponding author. Systems Analysis Laboratory, Helsinki University of Technology, P.O. Box 1100, FIN02015 HUT, Finland. E-mail: erkka.nasakkala@hut.fi

2 Department of Industrial Economics and Technology Management, Norwegian University of Science and Technology, NO-7491 Trondheim, Norway. E-mail: Stein-Erik.Fleten@iot.ntnu.no
} 


\section{Introduction}

We study flexibility in choice of technology regarding investments in gas fired power plants. The model is motivated by the advances in combined cycle gas turbine (CCGT) technology, and the emergence of energy commodity markets worldwide. It has been customary for investors holding licenses to build such plants to plan as if the plants are to be run around the clock, year round. The reason for this is that gas is purchased on long-term physical takeor-pay contracts. The gas market is being liberalized, however, and in the future we expect a more market-oriented and flexible gas supply system.

The value of a gas fired power depends on the spark spread, defined as the difference between the price of electricity and the cost of gas used for the generation of electricity. The stochastic process governing the evolution of the spark spread can be inferred from electricity and gas forward prices. The cash flows of an operating gas fired power plant are given by the spark spread less nonfuel variable costs, emission costs and fixed costs. If the emission costs exceed the spark spread some losses can be avoided by ramping down the plant. When the plant is ramped down only fixed costs remain.

We assume that there are two technologies for a gas fired power plant. A base load plant produces electricity independent of the spark spread and emission costs. A peak load plant is ramped up and down according to price changes. A base load plant has a constant gas consumption, whereas the gas consumption of a peak load plant varies. Thus, the nonfuel operational costs of a base load plant are lower. There are various alternatives in the design of CCGT plants, e.g. the steam cycle can be modified. Often base load plants can be upgraded into peak load plants. We calculate thresholds for building and upgrading a base load plant. We also study how the investment decision is changed when there is a possibility to build a non-upgradeable base load plant. The upgradeable and non-upgradeable base load plants are mutually exclusive alternatives.

The spark spread based valuation of power plants is studied in Deng, Johnson, and Sogomonian (2001). He and Pindyck (1992) study the output flexibility for a firm having two possible products to produce. Brekke and Schieldrop (2000) study the input flexibility in a 
power plant when the plant can be either gas or oil fired. Often the commodity prices are described by geometric Brownian motion. A number of authors have argued that meanreverting models are more appropriate for commodities (see, e.g., Laughton and Jacoby 1993 and 1995, Cortazar and Schwartz, 1994, and Smith and McCardle, 1999). Schwartz and Smith (2000) develop a two-factor model where the short-term deviations are modeled with a meanreverting process and the equilibrium price evolves according to a Brownian motion. We use similar two-factor model for the spark spread. The short-term deviations reflect non-persistent changes, for example, changes in demand resulting from variations in the weather or in the current supply system. The equilibrium price reflects fundamental changes that are expected to persist, for example, expectations of the discovery of natural gas. Other two-factor models with long- and short-term factors include, among others, Ross (1997) and Pilipović (1998).

Our results indicate that selecting a plant type which is first profitable leads to a non-optimal investment policy. The investment option value must be calculated for the maximum value of all possible projects. This result applies to the situation of choosing among mutually exclusive projects more generally. It is customary to think that uncertainty postpones investment decisions (see, e.g., Dixit and Pindyck, 1994). In this particular case increase in short-term volatility makes the investments to peak load plants more attractive and thus preempts investment to such plants.

The paper is organized as follows. We derive the basic valuation formulas in Section 2 . Boundary conditions are used to solve the model in Section 3. The model is illustrated with an example in Section 4. Section 5 concludes the study.

\section{$2 \quad$ Mathematical model}

Our analysis is based on electricity and gas forward prices. In describing the probabilistic structure of electricity and gas markets, we will refer to a probability space $(\Omega, \mathbb{F}, P)$, where $\Omega$ is a set, $\mathbb{F}$ is a $\sigma$-algebra of subsets of $\Omega$, and $P$ is a probability measure on $\mathbb{F}$. We denote the $T$-maturity forward prices, at time $t$, on electricity by $S_{e}(t, T)$ and on gas by $S_{g}(t, T)$. By allowing $T$ to vary from $t$ to $\tau$ we get forward curves $S_{e}(t, \cdot):[t, \tau] \rightarrow \mathbf{R}_{+}$and $S_{g}(t, \cdot):[t, \tau] \rightarrow \mathbf{R}_{+}$. Seasonality in electricity and gas supply and demand combined with limited storage opportunities causes cycles and peaks in the forward curves. 


\subsection{Spark spread process}

Spark spread is defined as the difference between the price of electricity and the cost of gas used for the generation of electricity. Thus, the $T$-maturity forward price on spark spread is

$$
S(t, T)=S_{e}(t, T)-K_{H} S_{g}(t, T),
$$

where heat rate $K_{H}$ is the amount of gas required to generate $1 M W h$ of electricity. Heat rate measures the efficiency of the gas plant: the lower the heat rate, the more efficient the facility. The efficiency of a gas fired power plant varies slightly over time and with the output level. Still, the use of a constant heat rate is considered plausible for long-term analyses (see, e.g., Deng, Johnson, and Sogomonian, 2001). Note that the value of the spark spread can be negative as well as positive.

When the electricity and gas derivative markets are free of arbitrage, the value of all derivative instruments are given by the expected value of future cash flows with respect to an equivalent martingale measure $Q$ (see e.g. Schwartz, 1997). Since electricity and gas are nonstorable commodities their expected returns under $Q$ are not usually equal to the risk-free rate, but the electricity and gas forwards are financial assets, and thus their expected returns under $\mathrm{Q}$ are equal to the risk-free interest rate. As the value of a forward contract when initiated is defined to be zero, the $T$-maturity forward price on spark spread at time $t$ is

$$
S(t, T)=E^{Q}\left[S(T) \mid F_{t}\right]
$$

where $S(T)$ is the spark spread at time $T$. Thus, the dynamics of the spark spread process under the pricing measure $Q$ can be can be inferred from forward prices. The seasonality in electricity and gas forward curves have similar characteristics, as electricity and gas are often used to same purposes, such as heating. Thus, the seasonality in electricity and gas forward curves decays from the spark spread forward curve. Motivated by this, we ignore the seasonality in the spark spread. We give an example process of spark spread in Section 4. The following assumption describes the dynamics of the spark spread process.

ASSUMPTION 1. The spark spread is a sum of short-term deviations and equilibrium price

$$
S(t)=\chi(t)+\xi(t)
$$

where the short-term deviations $\chi(\cdot)$ are assumed to revert toward zero following an OrnsteinUhlenbeck process 


$$
d \chi(t)=-\kappa \chi(t) d t+\sigma_{\chi} d B_{\chi}(t)
$$

and the equilibrium price $\xi(\cdot)$ is assumed to follow a Brownian motion process

$$
d \xi(t)=\mu_{\xi} d t+\sigma_{\xi} d B_{\xi}(t)
$$

where $\kappa, \sigma_{\chi}, \mu_{\xi}$, and $\sigma_{\xi}$ are constants. $B_{\kappa}(\cdot)$ and $B_{\xi}(\cdot)$ are standard Brownian motions, with correlation $\rho d t=d B_{\chi} d B_{\xi}$ on the probability space $(\Omega, F, Q)$, along with standard filtration $\left\{F_{t}: t \in[0, T]\right\}$.

When the spark spread increases, high cost producers will enter the market putting downward pressure on prices. Conversely, when prices decrease some high cost producers will withdraw capacity temporarily, putting upward pressure on prices. As these entries and exits are not instantaneous, prices may be temporarily high or low, but will tend to revert toward equilibrium price $\xi(\cdot)$. The mean-reversion parameter $\kappa$ describes the rate at which the short-term deviations $\chi(\cdot)$ are expected to decay. The uncertainty in the equilibrium price is caused by the uncertainty in the fundamental changes that are expected to persist. For example, advances in gas exploration and production technology, changes in the discovery of natural gas, improved gas fired power plant technology, and political and regulatory effects can cause changes to the equilibrium price. Other models where the two-factors are interpreted as short- and long-term factors are, for example, Schwartz and Smith (2000), Ross (1997), and Pilipović (1998). The decreasing forward volatility structure typical for commodities can be seen as a consequence of mean-reversion in commodity spot prices (see, e.g., Schwartz, 1997).

The following corollary states the distribution of the future spark spread values.

COROLLARY 1. When the spark spread has dynamics given in (3)-(5) the prices are normally distributed, and the expected value and variance are given by

$$
\begin{aligned}
& E\left[S(T) \mid F_{t}\right]=\mathrm{e}^{-\kappa(T-t)} \chi(t)+\xi(t)+\mu_{\xi}(T-t) \\
& \operatorname{Var}(S(T))=\frac{\sigma_{\chi}^{2}}{2 \kappa}\left(1-\mathrm{e}^{-2 \kappa(T-t)}\right)+\sigma_{\xi}^{2}(T-t)+2\left(1-\mathrm{e}^{-\kappa(T-t)}\right) \frac{\rho \sigma_{\chi} \sigma_{\xi}}{\kappa}
\end{aligned}
$$

PROOF: See, e.g., Schwartz and Smith (2000). 
The short-term deviations in the expected value decrease exponentially as a function of maturity, whereas the equilibrium price changes linearly. The time in which a short-term deviation is expected to halve is given by

$$
T_{1 / 2}=-\frac{\ln (0.5)}{\kappa}
$$

Also the spark spread variance decreases as a function of mean-reversion $\kappa$. Neither the short-term deviations $\chi(t)$ nor the equilibrium price $\xi(t)$ are directly observable from market quotas, but they can be estimated from forward prices. Intuitively, the long-maturity forwards give information of the equilibrium price, whereas the short-term dynamics can be estimated from the short-maturity forwards. The estimation of the spark spread process parameters will be considered in Section 4. As the spark spread values are normally distributed the values can be negative as well as positive.

\subsection{Plant values}

The following assumption characterizes the two technologies for a gas fired power plant.

ASSUMPTION 2. A base load plant produces electricity with constant capacity. A peak load plant can be ramped up and down without delay. The costs associated with starting up a peak load plant can be amortized into fixed costs.

A base load plant will produce electricity even if it turns to be unprofitable, whereas a peak load plant can be ramped down whenever it is unprofitable to use the plant. In a gas fired power plant, the operation and maintenance costs do not vary much over time and the response times are in the order of several hours. Thus, we are assuming that the peak load plant is more flexible than it really is, but for efficient plants the error in the long-term valuation analysis will be small (see, e.g., Deng and Oren, 2003). The inflexibility of a base load plant can be caused by constraints in the gas inflow. If a peak load plant is ramped down, but the gas inflow cannot be changed the gas will be lost. Thus, it is never optimal to ramp down a plant with rigid gas supply. Such delivery agreements are typically part of the take-or-pay contract that the power plant needs to secure its physical gas deliveries.

For simplicity, we assume that the (nonfuel) operational costs are deterministic, i.e. they can be amortized into investment costs. We use a constant risk free interest rate $r$. For every unit of electrical energy generated, the plant must pay environmental taxes due to emission of 
greenhouse gases and other pollutants. This is represented by the emission cost $E$. The following lemma gives the value of a base load plant.

LEMMA 1. The base load plant value, at time $t$, is

$$
V_{1}(\chi, \xi)=\bar{C}\left(\frac{\chi(t)}{\kappa+r}+\frac{\xi(t)-E}{r}+\frac{\mu_{\xi}}{r^{2}}-\mathrm{e}^{-r(\bar{T}-t)}\left(\frac{\mathrm{e}^{-\kappa(\bar{T}-t)} \chi(t)}{\kappa+r}+\frac{\xi(t)-E}{r}+\frac{\mu_{\xi}(r(\bar{T}-t)+1)}{r^{2}}\right)\right)
$$

where $\bar{T}$ is the lifetime of the plant, and $\bar{C}$ is the capacity of the plant.

PROOF: The base load plant value is the present value of expected operating cash flows, i.e.,

$$
\begin{aligned}
V_{1}(\chi, \xi) & =\bar{C} \int_{t}^{\bar{T}} e^{-r(s-t)}\left(E\left[S(s) \mid F_{t}\right]-E\right) d s= \\
& =\bar{C} \int_{t}^{\bar{T}} e^{-r(s-t)}\left(e^{-\kappa(s-t)} \chi(t)+\xi(t)-E+\mu_{\xi}(s-t)\right) d s
\end{aligned}
$$

integration gives (9).

Q.E.D.

The base load plant value is affected by the expected value of spark spread, but not by the short-term and equilibrium volatility parameters $\sigma_{\chi}$ and $\sigma_{\xi}$. A base load plant cannot exploit unexpected changes in the spark spread, thus the value of a base load plant is just the discounted sum of expected spark spread values less emission costs.

A peak load plant operates only when the spark spread exceeds emission costs. The following lemma gives the value of a peak load plant.

LEMMA 2. The peak load plant value, at time $t$, is

$$
V_{2}(\chi, \xi)=\bar{C} \int_{t}^{\bar{T}} e^{-r(s-t)}\left(\frac{v(s)}{\sqrt{2 \pi}} e^{\left(-\frac{(E-\mu(s))^{2}}{2 v^{2}(s)}\right)}+(\mu(s)-E)\left(1-\Phi\left(\frac{E-\mu(s)}{v(s)}\right)\right)\right) d s
$$

where $\Phi(\cdot)$ is the normal cumulative distribution function. The expected value $\mu(s)=E\left[S(s) \mid F_{t}\right]$ and variance $v^{2}(s)=\operatorname{Var}(S(s))$ for the spark spread are given with Corollary 1.

PROOF: See Appendix A.

A peak load plant value increases as a function of the spark spread variance, as an owner of a peak load plant can avoid losses caused by unexpected changes in the spark spread by 
ramping down the plant. The peak load plant value is the discounted sum of expected spark spread values less emission costs plus the option value of being able to ramp down.

\subsection{Investment options}

The following assumption characterizes the variables affecting the building and upgrading decision.

ASSUMPTION 3. The thresholds for building and upgrading are calculated for the equilibrium price assuming that the current short-term deviation is zero. When the investment decisions are made it is assumed that the lifetime of the plant is infinite.

Assumption 3 states that when the investments in gas fires power plants are considered the investment decisions are made as a function of the equilibrium price $\xi(\cdot)$. Thus, neither building nor upgrading is started due to the current realization of short-term deviations. The short-term dynamics still affect the value of a peak load plant, and thus they also affect the building and upgrading decisions. In other words, the short-term dynamics are important in the investment decisions, even though their current realization is ignored when the investment decisions are made. The omission of the current short-term realization is motivated by the fact that gas fired power plants are long-term investments, and thus a gas plant investment is never commenced due to the current spike in the short-term deviations. This is realistic as long as the expected lifetime of the short-term deviations is considerably smaller than the expected lifetime of the plant. In Section 4 we estimate that, in our example data, the mean-reversion parameter $\kappa$ is 8.1. Equation (8) gives that, in this case, short-term variations are expected to halve in about one month. Usually, the life time of a gas fired power plant is assumed to be around 25 years. Thus, the ignorance of the current short-term realization is realistic. The infinite lifetime assumption is motivated by the fact that the plant's lifetime is often increased by upgrading and reconstructions.

The following lemma gives the value of the build option when it is not optimal to exercise the option (i.e., when $\xi<\xi_{H 0}$ ).

LEMMA 3. The value of an option to build a base load plant is

$$
F_{0}(\xi)=A_{1} e^{\beta_{1} \xi} \quad \text { when } \quad \xi<\xi_{H 0}
$$


where $A_{1}$ is a positive parameter. Parameter $\beta_{1}$ is given by

$$
\beta_{1}=\frac{-\mu_{\xi}+\sqrt{\mu_{\xi}^{2}+2 \sigma_{\xi}^{2} r}}{\sigma_{\xi}^{2}}>0 .
$$

PROOF: See Appendix B.

The build option value increases exponentially as a function of the equilibrium price. The parameter $A_{1}$ depends on what type of plant is optimal to built. Method to solve the parameter $A_{1}$ will be studied in the next section. When a decision to build an upgradeable base load plant is made the investor receives both the upgradeable base load plant and the option to upgrade the base load plant. The upgrade option value is given by the following lemma when it is not optimal to exercise the upgrade option (i.e., when $\xi>\xi_{H 1}$ )

LEMMA 4. The value of an option to upgrade a base load plant into a peak load plant is

$$
F_{1}(\xi)=D_{2} e^{\beta_{2} \xi} \quad \text { when } \quad \xi>\xi_{H 1}
$$

where $D_{2}$ is a positive parameter. Parameter $\beta_{2}$ is given by

$$
\beta_{2}=\frac{-\mu_{\xi}-\sqrt{\mu_{\xi}^{2}+2 \sigma_{\xi}^{2} r}}{\sigma_{\xi}^{2}}<0 .
$$

PROOF: The proof is similar to that of the build option (Appendix A) but now the option becomes less valuable as the spark spread increases. On the other hand, it becomes more valuable as spark spread decreases.

Q.E.D.

The upgrade option value decreases exponentially as a function of the equilibrium price. The parameter $D_{2}$ depends on the difference of plant values and upgrading costs. Method to solve the parameter $D_{2}$ will be studied in the next section. The value of an upgradeable base load plant's owner is the value of a base load plant plus an option to upgrade (i.e. $\left.V_{1}(\chi, \xi)+F_{1}(\xi)\right)$, whereas the value of a non-upgradeable base load plant's owner is $V_{1}(\chi, \xi)$. The values will be illustrated with an example in Section 4.

\section{Solution method}

In this section we compute the optimal investment strategy. First, we consider investment in an upgradeable base load plant. We compute optimal thresholds for building and upgrading. 
Second, we assume that there is also an opportunity to build a non-upgradeable base load plant. In this case both timing and optimal investment type will be decided.

The holder of a license to build a power plant does nothing as long as the equilibrium price is below building threshold $\xi_{H 0}$. It is optimal to exercise the build option when the option value becomes equal to the values gained by exercising the option, i.e.

$$
F_{0}\left(\xi_{H 0}\right)=V_{1}\left(0, \xi_{H 0}\right)+F_{1}\left(\xi_{H 0}\right)-I
$$

where $I$ is the investment cost of an upgradeable base load plant. Once a base load plant is built the owner has a base load plant, whose value is $V_{1}(\chi, \xi)$, and an option to upgrade the base load plant into a peak load plant. The owner of an upgradeable base load plant does nothing as long as the equilibrium price is above upgrading threshold $\xi_{H 1}$. An upgrade is commenced when the upgrade option value is equal to values gained by upgrading the base load plant. The values gained are the difference of peak and base load plant values minus upgrade cost $\Delta I$. Thus, upgrading is optimal when

$$
F_{1}\left(\xi_{H 1}\right)=V_{2}\left(0, \xi_{H 1}\right)-V_{1}\left(0, \xi_{H 1}\right)-\Delta I \text {. }
$$

Also the smooth-pasting conditions must hold when the options are exercised (for an intuitive proof see, e.g., Dixit and Pindyck, 1994 and for a rigorous derivation see Samuelson, 1965)

$$
\begin{aligned}
& \frac{\partial F_{0}\left(\xi_{H 0}\right)}{\partial \xi}=\frac{\partial V_{1}\left(0, \xi_{H 0}\right)}{\partial \xi}+\frac{\partial F_{1}\left(\xi_{H 0}\right)}{\partial \xi} \\
& \frac{\partial F_{1}\left(\xi_{H 1}\right)}{\partial \xi}=\frac{\partial V_{2}\left(0, \xi_{H 1}\right)}{\partial \xi}-\frac{\partial V_{1}\left(0, \xi_{H 1}\right)}{\partial \xi} .
\end{aligned}
$$

Equations (16)-(19) give four equations for four unknowns $\left(\xi_{H 1}, \xi_{H 0}, A_{1}, D_{2}\right)$. The equations cannot be solved analytically, but a numerical solution can be attained. The properties of the solution will be discussed in the example Section 4 .

So far we have studied a base load plant upgradeable into a peak load plant. Next we consider the case where there is a choice between an upgradeable and non-upgradeable base load plant. It is more expensive to build and operate an upgradeable plant, and we let $I_{0}$ represent the investment cost of a non-upgradeable base load plant. 
There are two mutually exclusive investments opportunities: the option to invest in an upgradeable plant, and the option to invest in a non-upgradeable plant. It is optimal to exercise the build option when the most valuable investment opportunity becomes profitable. Thus, the optimal plant type and the building threshold are chosen by

$$
\max \left(V_{1}\left(0, \xi_{H 0}\right)+F_{1}\left(\xi_{H 0}\right)-I, V_{1}\left(0, \xi_{H 0}\right)-I_{0}\right)=F_{0}\left(\xi_{H 0}\right),
$$

which can be written as

$$
\max \left(F_{1}\left(\xi_{H 0}\right)-I,-I_{0}\right)=F_{0}\left(\xi_{H 0}\right)-V_{1}\left(0, \xi_{H 0}\right) .
$$

From (21) it follows that if the solution of (16)-(19) satisfies

$$
F_{1}\left(\xi_{H 0}\right)>I-I_{0}
$$

the upgradeable choice is optimal. The extra investment cost associated with choosing an upgradeable plant are $I-I_{0}$, which can be interpreted as the cost of acquiring the upgrade option. If the upgrade option is worth more than its cost then the upgradeable plant is chosen. If the threshold $\xi_{H 0}$ is very high, the option to upgrade will be of little value, and an inflexible plant will be chosen. If upgrading is cheap (i.e. $I-I_{0}$ is low) it is more likely that an upgradeable plant will be chosen.

If condition (22) is not satisfied the non-upgradeable base load plant is optimal. When the non-upgradeable choice is optimal there is no upgrading threshold and the value matching and smooth-pasting conditions are

$$
\begin{aligned}
& I_{0}+F_{0}\left(\xi_{H 0}\right)=V_{1}\left(0, \xi_{H 0}\right) \\
& \frac{\partial F_{0}\left(\xi_{H 0}\right)}{\partial \xi}=\frac{\partial V_{1}\left(0, \xi_{H 0}\right)}{\partial \xi} .
\end{aligned}
$$

The following proposition summarizes the investment decision.

PROPOSITION 1. For an upgradeable plant the build and upgrade thresholds $\xi_{H 0}$ and $\xi_{H 1}$ as well as the option values, i.e. parameters $A_{1}$ and $D_{2}$, are given by

$$
\begin{aligned}
& F_{0}\left(\xi_{H 0}\right)+I=V_{1}\left(0, \xi_{H 0}\right)+F_{1}\left(\xi_{H 0}\right), \\
& F_{1}\left(\xi_{H 1}\right)+\Delta I=V_{2}\left(0, \xi_{H 1}\right)-V_{1}\left(0, \xi_{H 1}\right) . \\
& \frac{\partial F_{0}\left(\xi_{H 0}\right)}{\partial \xi}=\frac{\partial V_{1}\left(0, \xi_{H 0}\right)}{\partial \xi}+\frac{\partial F_{1}\left(\xi_{H 0}\right)}{\partial \xi} \\
& \frac{\partial F_{1}\left(\xi_{H 1}\right)}{\partial \xi}=\frac{\partial V_{2}\left(0, \xi_{H 1}\right)}{\partial \xi}-\frac{\partial V_{1}\left(0, \xi_{H 1}\right)}{\partial \xi} .
\end{aligned}
$$


When there is a possibility to build a non-upgradeable plant, if the solution of (25)-(28) satisfies

$$
F_{1}\left(\xi_{H 0}\right)>I-I_{0}
$$

the upgradeable plant is optimal. If condition (29) is not satisfied the non-upgradeable plant is optimal and the building threshold and the build option value are given by

$$
\begin{aligned}
& I_{0}+F_{0}\left(\xi_{H 0}\right)=V_{1}\left(0, \xi_{H 0}\right) \\
& \frac{\partial F_{0}\left(\xi_{H 0}\right)}{\partial \xi}=\frac{\partial V_{1}\left(0, \xi_{H 0}\right)}{\partial \xi} .
\end{aligned}
$$

PROOF: Follows directly from (16)-(24).

Q.E.D.

Qualitative properties of the solution will be discussed in the context of an example in the next section.

\section{Example}

In this section we illustrate our model with a simple example. The example consists of three parts. First, we introduce the data, including methods to estimate the parameters. Second, we calculate the threshold and option values with our parameter estimates. In part three we analyze the sensitivity of the results to some key parameters.

We study building of a gas fired combined cycle power plant in Northern Europe, thus we use electricity data from Nord Pool (The Nordic Power Exchange) and gas data from International Petroleum Exchange (IPE). We use daily forward quotas from the years 19982002. The spread process is calculated from electricity and gas prices by adjusting the gas prices with the heat rate so that a unit of gas corresponds to $1 M W h$ of electricity generated. The efficiency of a combined cycle gas fired turbine is estimated to be $58.1 \%$, thus the heat rate $K_{H}$ is 1.72 . The currency used in Nord Pool is Norwegian krone (NOK). We use an exchange rate of $7 \mathrm{NOK} / \$$.

We use Kalman filtering techniques (see, e.g., Harvey, 1989 and West and Harrison 1996) to estimate the volatility and mean-reversion parameters from the short-maturity (seasonal 
contracts with 1-year maturity) forwards. The Kalman filter facilitates the calculation of the likelihood of observing a particular data series given a particular set of model parameters, thus we use maximum likelihood method to estimate the volatility and mean reversion parameters, i.e. $\kappa, \sigma_{\chi}$, and $\sigma_{\xi}$. For more about the estimation procedure see Schwartz and Smith (2000). The equilibrium drift $\mu_{\xi}$ is estimated with linear regression from long-maturity forward prices. In Figure 1 the seasonal quotas used for the volatility and mean-reversion estimation are illustrated together with the expected value and $68 \%$ confidence interval for the years 2002-2008. The expected value and confidence intervals are given by Corollary 1 .

[Figure 1 about here]

We assume that the $\mathrm{CO}_{2}$ emission costs are $8 \$$ ton and ignore possible uncertainty regarding future levels (or whether such taxes will be introduced at all). The uncertainty in emission costs could be modeled by increasing the uncertainty in the equilibrium price. The $\mathrm{CO}_{2}$ production of a combined cycle gas fired power plant is $363 \mathrm{~kg} / \mathrm{MWh}$, thus an $\mathrm{CO}_{2}$ emission cost of $8 \$ /$ ton corresponds $20.3 \mathrm{NOK} / \mathrm{MWh}$. Our estimates for the spread process parameters and emission cost are summarized in Table 1. The risk-free interest rate is $6 \%$.

Table 1: Spark spread parameters

\begin{tabular}{l|lllllll} 
Parameter & $\kappa$ & $E$ & $\mu_{\xi}$ & $\rho$ & $\sigma_{\chi}$ & $\sigma_{\xi}$ & $r$ \\
\hline Unit & & $N O K / M W h$ & $N O K / M W h$ & & $N O K / M W h$ & $N O K / M W h$ & \\
\hline Value & 8.1 & 20.8 & 0.2 & -0.3 & 42.1 & 9.6 & $6 \%$
\end{tabular}

We study a gas plant whose maximum capacity is $415 \mathrm{MW}$. The utilization rate of the plant is approximately $90 \%$. Thus, the production capacity is $\bar{C}=3.27 \mathrm{GWh} /$ year. It is estimated that building an upgradeable base load plant costs about 1620 MNOK. The maintenance costs for such a plant are approximately 50 MNOK/year. By summing the building costs and discounted maintenance costs we get that the investment costs are $2450 \mathrm{MNOK}$. The costs to upgrade a base load plant into a peak load plant are about $5 \%$ of the investment costs, i.e. $\Delta I=122.5$ MNOK. A non-upgradeable base load plant is cheaper to build and operate. We assume that the investment costs of a non-upgradeable plant are $1 \%$ lower than for an 
upgradeable plant, i.e. $I_{0}=2425.5$ MNOK. The cost estimates are based on Undrum, Bolland, Aarebrot (2000). The gas plant parameters are summarized in Table 2.

Table 2: The gas plant parameters

\begin{tabular}{l|llll} 
Parameter & $\bar{C}$ & $I$ & $\Delta I$ & $I_{0}$ \\
\hline Unit & GWh/year & MNOK & MNOK & MNOK \\
\hline Value & 3.27 & 2450 & 122.5 & 2425.5
\end{tabular}

The plant and option values as a function of equilibrium price for the upgradeable plant are illustrated in Figure 2. Also the investment thresholds are given. The thresholds and option values are given by Proposition 1. In Figure 2 the grey lines are the option values. The dashed gray line is the upgrade option and the solid gray line is the build option. The plant values are illustrated with the black lines. The solid black line is the peak load plant and the dashed black line is the base load plant. Naturally, the peak load plant is always more valuable than the base load plant.

[Figure 2 about here]

In the upper picture of Figure 3 the value of an upgradeable base load plant's owner $V_{1}(0, \xi)+F_{1}(\xi)$ is compared with the value of a non-upgradeable plant's owner $V_{1}(0, \xi)$. The owner of an upgradeable plant is always more valuable, but the difference becomes smaller and smaller as the spark spread increases. In the lower picture of Figure 3 the adjusted present value of the upgradeable base load plant, i.e. $V_{2}(0, \xi)-V_{1}(0, \xi)-F_{1}(\xi)-\Delta I$, is presented. Adjusted present value of the upgradeable plant is the value gain obtained by upgrading a base load plant into a peak load plant. As spark spread decreases, and approaches the upgrade trigger $\xi_{H 1}$ the adjusted present value, $V_{2}(0, \xi)-V_{1}(0, \xi)-F_{1}(\xi)-\Delta I$, approaches zero from below. Upgrading commences when adjusted present value equals zero.

[Figure 3 about here]

Proposition 1 states that it is optimal to build an upgradeable base load plant when the value of the build option at the building threshold is greater than 49 MNOK (i.e. $F_{1}\left(\xi_{H 0}\right)>2450$ - 
$2401 \mathrm{MNOK})$. In Figure 2 the value of the upgrade option at the building threshold is $F_{1}\left(\xi_{H 0}\right)=43.5 \mathrm{MNOK}$, thus it optimal to build a non-upgradeable plant if there is a possibility to build such a plant. The building threshold for a non-upgradeable plant is given by Proposition 1. The thresholds for different types of plants are summarized in Table 3.

Table 3: Thresholds

\begin{tabular}{l|ll|l} 
& \multicolumn{2}{|c|}{ upgradeable } & non-upgradeable \\
\hline Variable & $\xi_{H 0}^{U}$ & $\xi_{H 1}$ & $\xi_{H O}^{N}$ \\
\hline Unit & $N O K / M W h$ & $N O K / M W h$ & $N O K / M W h$ \\
\hline Value & 90.2 & 23.2 & 91.2
\end{tabular}

The building threshold for the non-upgradeable plant $\xi_{H O}^{N}$ is greater than the building threshold for the upgradeable plant $\xi_{H 0}^{U}$, even though the non-upgradeable plant is optimal choice. In this example case, the investment opportunity that first becomes profitable, when the equilibrium price reaches that level from below, is not optimal. In other words, it is not optimal to invest in the project with the lowest threshold.

In Figure 4 we illustrate the adjusted present values for both types of base load plants. The black dashed line is the adjusted present value, $V_{1}(0, \xi)+F_{1}(\xi)-F_{0}^{U}(\xi)-I$, of an investment without the possibility to build a non-upgradeable plant. The adjusted present value of an opportunity to build a non-upgradeable plant, $V_{1}(0, \xi)-F_{0}^{N}(\xi)-I_{0}$, is given by the solid black line. Note that the adjusted present value of an investment without the possibility to build a non-upgradeable plant can be higher than the adjusted present value of an investment with the possibility to choose between the two possibilities. Thus, the optimal plant type cannot either be decided by considering the adjusted present values.

[Figure 4 about here]

Let us analyze the sensitivity of the investment decision when there are the two possibilities. In Figure 5 the thresholds as a function of the upgrade costs $\Delta I$ are illustrated. The black 
lines are the thresholds for an upgradeable plant, while the gray lines are for non-upgradeable plant. The solid lines represent the optimal choice. In Figure 5 it is optimal to build an upgradeable plant when the upgrade costs $\Delta I$ are below 35 MNOK. The building threshold for non-upgradeable plant does not change when the upgrade costs are changed because upgrade costs do not affect the value of a non-upgradeable base load plant. The building threshold for an upgradeable plant is only slightly affected by the changes in the upgrade costs, whereas the upgrading threshold is more sensitive. Also Figure 5 indicates that when the project with lowest threshold is not optimal.

[Figure 5 about here]

In Figure 6 the thresholds as a function of equilibrium uncertainty $\sigma_{\xi}$ are illustrated. It is optimal to build a non-upgradeable plant when the equilibrium volatility $\sigma_{\xi}$ is below 10 $N O K / M W h$. Thus, if the equilibrium volatility $\sigma_{\xi}$ increases from current value 9.6 to 10 it is optimal to build an upgradeable plant and the building threshold increases to 92.4 $N O K / M W h$. In Figure 6 the building thresholds increase and the upgrade threshold decreases as a function of equilibrium volatility, i.e. uncertainty in equilibrium price postpones investment decisions.

[Figure 6 about here]

In Figures 7 and 8 we analyze the sensitivity of the thresholds to the short-term dynamics of the spark spread. In Figure 7 the thresholds as a function of short-term volatility $\sigma_{\chi}$ are analyzed. In Figure 8 the sensitivity to the mean-reversion of short-term variations, i.e. $\kappa$, are illustrated. In both figures the building threshold for a non-upgradeable plant is the gray line, and the thresholds for the upgradeable plant are the black lines. Lemma 1 states that the value of a base load plant is not affected by the short-term variations in the spark spread and by Assumption 3 the investment decisions are made as a function of equilibrium price. Thus, the building threshold for a non-upgradeable plant does not change as the short-term dynamics of the spark spread are changed. In Figures 7 and 8 the optimal choice is indicated with a solid line. Thus, the changes in $\sigma_{\chi}$ and $\kappa$ do not make the upgradeable choice optimal. 
In Figure 7 increase in short-term volatility preempts the upgrade decision, whereas in Figure 6 increase in equilibrium volatility postpones the upgrading. In other words, short-term and equilibrium volatility have an opposite effect on the timing of upgrading. Increase in shortterm volatility increases the value of a peak load plant, and thus makes earlier upgrading more favorable. Correspondingly, increase in equilibrium volatility increases also the build and upgrade option values, and thus postpones the investment decision.

[Figure 7 about here]

Figure 8 indicates that increase in the mean reversion $\kappa$ postpones the upgrade decision. By Corollary 1 , increase in mean reversion $\kappa$ decreases the variance of the spread process, i.e. decreases the value of a peak load plant. Thus increase in the mean-reversion postpones the upgrade decision.

[Figure 8 about here]

\section{Conclusions}

We use real options theory for investments in gas fired power plants. Our analysis is based on electricity and gas forward prices. The decision maker is assumed to have an exclusive right to invest, for example in the form of a previously acquired license. We derive a method to compute thresholds for building and upgrading, when a base load plant can be upgraded into a peak load plant. We also analyze how the investment decision is changed when there is a possibility to build a non-upgradeable plant.

Our numerical example illustrates that when the option to build is valued the interaction of possible investment opportunities needs to be considered. By considering the options to invest independently, and selecting the project which is first profitable, the result is a non-optimal exercise policy of the investment option. This result applies of course to the situation of choosing among mutually exclusive projects more generally. The optimal decision rule regarding investment timing cannot be found just by considering the threshold boundaries or adjusted present values of the individual projects. One has to calculate the investment option value for the maximum value of all possible projects. 
In this particular case increase in short-term volatility preempts the upgrade decision, as the value of a peak load plant increases as a function of short-term volatility. Thus, the shortterm and equilibrium volatility have an opposite effect on the upgrade decision. This contradicts the traditional thinking in real options literature that uncertainty postpones the investment decisions. 


\section{References}

Brekke, K.A. and Schieldrop B. (1999) Investment in flexible technologies under uncertainty, in: M.J. Brennan and L. Trigeorgis, eds., Project Flexibility, Agency, and Competition: New Developments in the Theory of Real Options (Oxford University Press, New York), 34-49.

Cortazar, G., Schwartz E.S. (1994), The evaluation of commodity contingent claims, Journal of Derivatives 1 27-39

Deng, S.J., Johnson B., Sogomonian A. (2001) Exotic electricity options and the valuation of electricity generation and transmission assets, Decision Support Systems, (30) 3, pp. 383-392

Deng, S.J., Oren S.S. (2003) Valuation of Electricity Generation Assets with Operational Characteristics, Probability in the Engineering and Informational Sciences, forthcoming Dixit, A.K., Pindyck, R.S. (1994) Investment under Uncertainty, Princeton University Press Gibson, R., Schwartz E. S., (1990), Stochastic Convenience yield and the pricing of oil contingent claims, The Journal of Finance, 45, pp. 959-976

Harvey, A. C. (1989), Forecasting, Structural Time Series Models and the Kalman Filter, Cambridge University Press, Cambridge, U.K.

He, H., and Pindyck, R.S. (1992) Investment In Flexible Production Capacity, Journal of Economic Dynamics and Control 16, 575-599

Laughton, D.G., Jacoby H.D.,(1993), Reversion, timing options, and long-term decision making, Financial Management 33 pp. 225-240

Laughton, D.G., Jacoby H.D.,(1995), The effects of reversion on commodity projects of different length, L. Trigeorgis, ed. Real Options in Capital Investment: Models, Strategies and Applications. Praeger, Westport, CT. 185-205

Pilipović, D. (1998), Energy Risk: Valuing and Managing Energy Derivatives, McGraw-Hill Ross, S. (1997), Hedging long run commitments: Exercises in incomplete market pricing, Banca Monte Econom. 25 pp. 141-159

Samuelson, P.A. (1965) Rational theory of warrant pricing, Industrial Management Review 6, pp. 13-31

Schwartz, E.S. (1997) The Stochastic Behavior of Commodity Prices: Implications for Valuation and Hedging, The Journal of Finance, 52 (3), pp. 923-973 
Schwartz, E.S. (1998) Valuing Long-Term Commodity Assets, Financial Management, 27 (1), pp. $57-66$

Schwartz, E.S., Smiht J. E., (2000), Short-term Variations and Long-Term dynamics in Commodity Prices, Management Science, 46 (7), pp. 893-911

Smith, J.E., McCardle K.F., (1998), Valuing oil properties: Integrating option pricing and decision analysis approaches. Operations Research 46, pp. 1-15

Undrum, H., Bolland, O., and Aarebrot, E. (2000) Economical assessment of natural gas fired combined cycle power plant with $\mathrm{CO} 2$ capture and sequestration, presented at the Fifth International Conference on Greenhouse Gas Control Technologies, Cairns, Australia

West, M., Harrison J., (1996), Bayesian Forecasting and Dynamic Models 2nd ed. SpringerVerlag, New York

\section{Appendix A}

As a peak load plant operates only when the spark spread exceeds emission costs, the peak load plant, at time $s$, can be replicated with $\bar{C}$ European call options on the spark spread with strike price equal to the emission costs $E$. At time $t$, the value of such an option, maturing at time $s$, is

$$
c(s)=e^{-r(s-t)} E\left[\max (S(s)-E, 0) \mid F_{t}\right]=e^{-r(s-t)}\left(\int_{E}^{\infty}(y-E) h(y) d y\right)
$$

where $y$ is a normally distributed variable with mean $\mu(s)$ and variance $v^{2}(s)$. $h(y)$ is the density function of a normally distributed variable y. The integration gives

$$
c(s)=e^{-r(s-t)}\left(\frac{v(s)}{\sqrt{2 \pi}} e^{-\frac{(E-\mu(s))^{2}}{2 v^{2}(s)}}+(\mu(s)-E)\left(1-\Phi\left(\frac{E-\mu(s)}{v(s)}\right)\right)\right),
$$

where $\Phi(\cdot)$ is the normal cumulative distribution function. The value of a plant with lifetime $\bar{T}$ is given by

$$
V_{2}(\chi, \xi)=\bar{C} \int_{t}^{\bar{T}} c(s) d s
$$

which gives

$$
V_{2}(\chi, \xi)=\bar{C} \int_{t}^{\bar{T}} e^{-r(s-t)}\left(\frac{v(s)}{\sqrt{2 \pi}} e^{\left(-\frac{(E-\mu(s))^{2}}{2 v^{2}(s)}\right)}+(\mu(s)-E)\left(1-\Phi\left(\frac{E-\mu(s)}{v(s)}\right)\right)\right) d s
$$




\section{Appendix B}

When it is not optimal to exercise the build option (i.e., when $\xi<\xi_{H 0}$ ), the option to build $F_{0}$ must satisfy following Bellman equation

$$
r F_{0}(\xi) d t=E^{Q}\left[d F_{0}(\xi)\right] \text {, when } \xi<\xi_{H 0},
$$

where $E^{Q}$ is the expectation operator under the pricing measure $Q$. Itô's lemma gives following differential equation for the option value

$$
\frac{1}{2} \sigma^{2} \frac{\partial^{2} F_{0}(\xi)}{\partial^{2} S}+\alpha \frac{\partial F_{0}(\xi)}{\partial S}-r F_{0}(\xi)=0, \text { when } \xi<\xi_{H 0}
$$

A solution to a differential equation is a linear combination of two independent solutions. Thus, the value of the option to build is

$$
F_{0}(\xi)=A_{1} \mathrm{e}^{\beta_{1} \xi}+A_{2} \mathrm{e}^{\beta_{2} \xi}, \text { when } \xi<\xi_{H 0},
$$

where $A_{1}, A_{2}$ are unknown parameters and $\beta_{1}$ and $\beta_{2}$ are the roots of the fundamental quadratic equations given by

$$
\begin{aligned}
& \beta_{1}=\frac{-\mu_{\xi}+\sqrt{\mu_{\xi}^{2}+2 \sigma_{\xi}^{2} r}}{\sigma_{\xi}^{2}}>0 \\
& \beta_{2}=\frac{-\mu_{\xi}-\sqrt{\mu_{\xi}^{2}+2 \sigma_{\xi}^{2} r}}{\sigma_{\xi}^{2}}<0 .
\end{aligned}
$$

The value of the option to build approaches zero as the spark spread decreases (i.e. $A_{2}=0$ ), and thus

$$
F_{0}(\xi)=A_{1} \mathrm{e}^{\beta_{1} \xi}, \text { when } \xi<\xi_{H 0}
$$

\section{Figures}

Figure 1: Example realization of spread process and a future estimate

Figure 2: Plant and option values

Figure 3: Values of plants' owners and incremental value of the upgradeable base load plant

Figure 4: Adjusted present values of different investors

Figure 5: Thresholds as a function of upgrade costs

Figure 6: Thresholds as a function of equilibrium volatility

Figure 7: Thresholds as a function of short-term volatility

Figure 8: Thresholds as a function of mean-reversion 


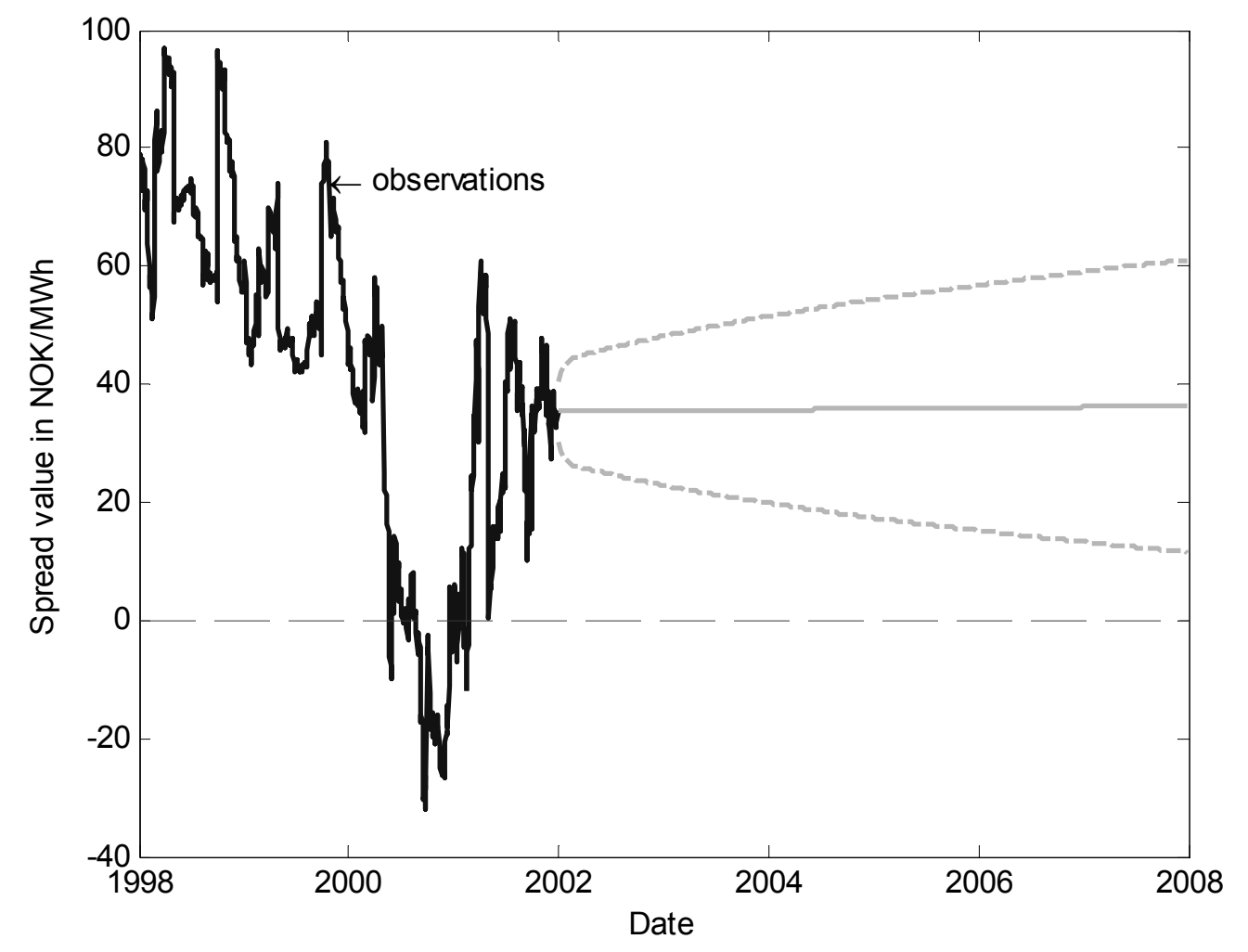

Figure 1 


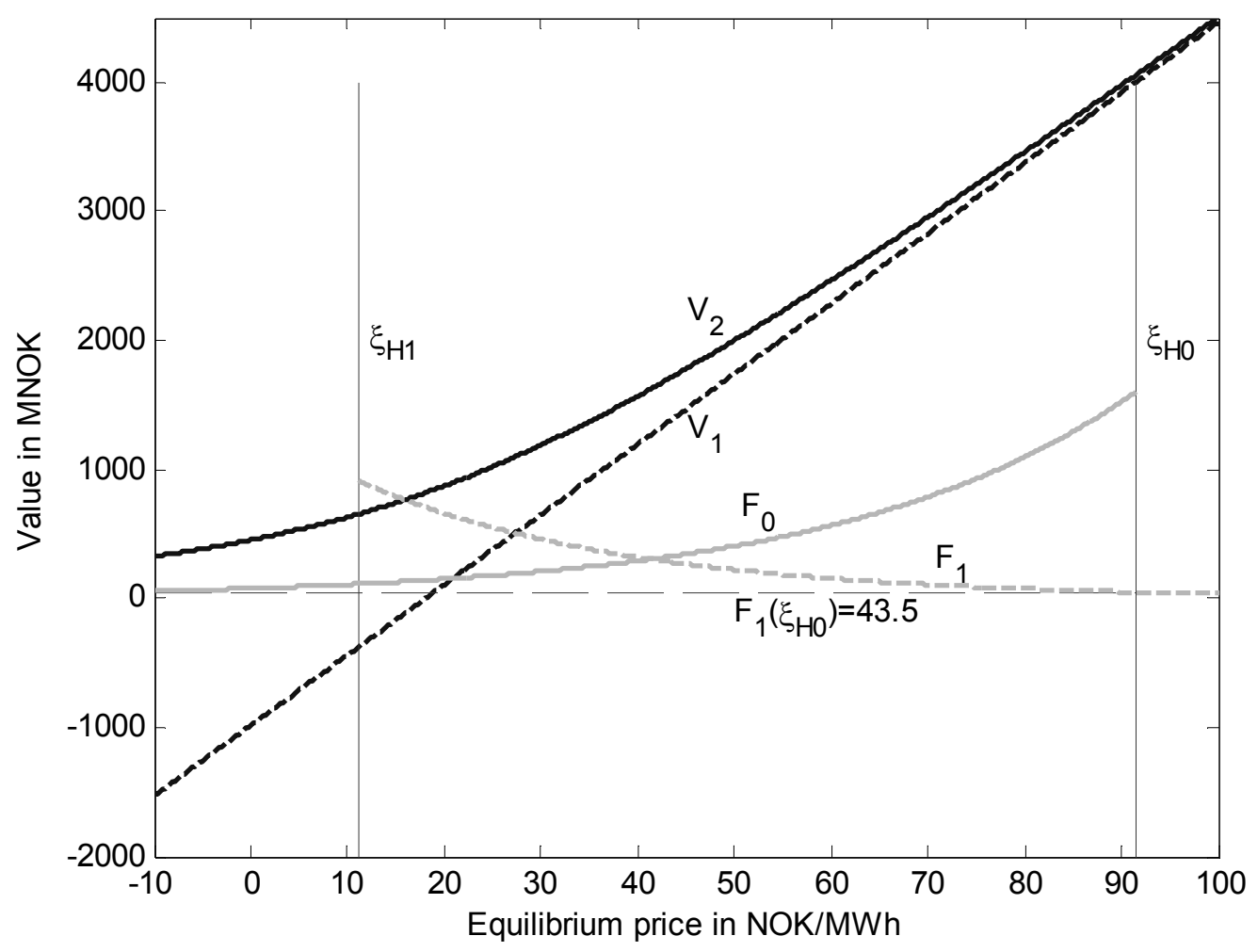

Figure 2
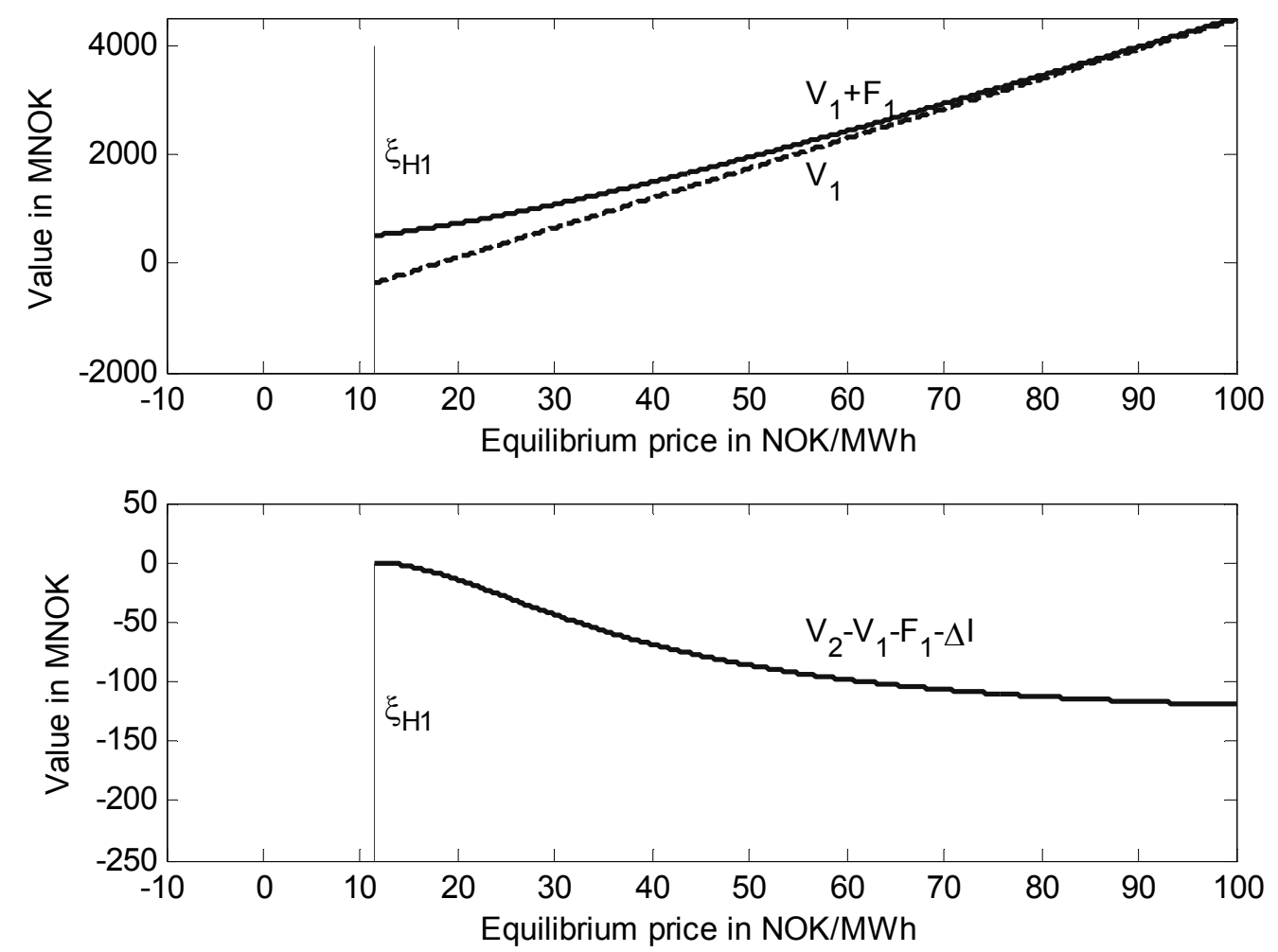

Figure 3 


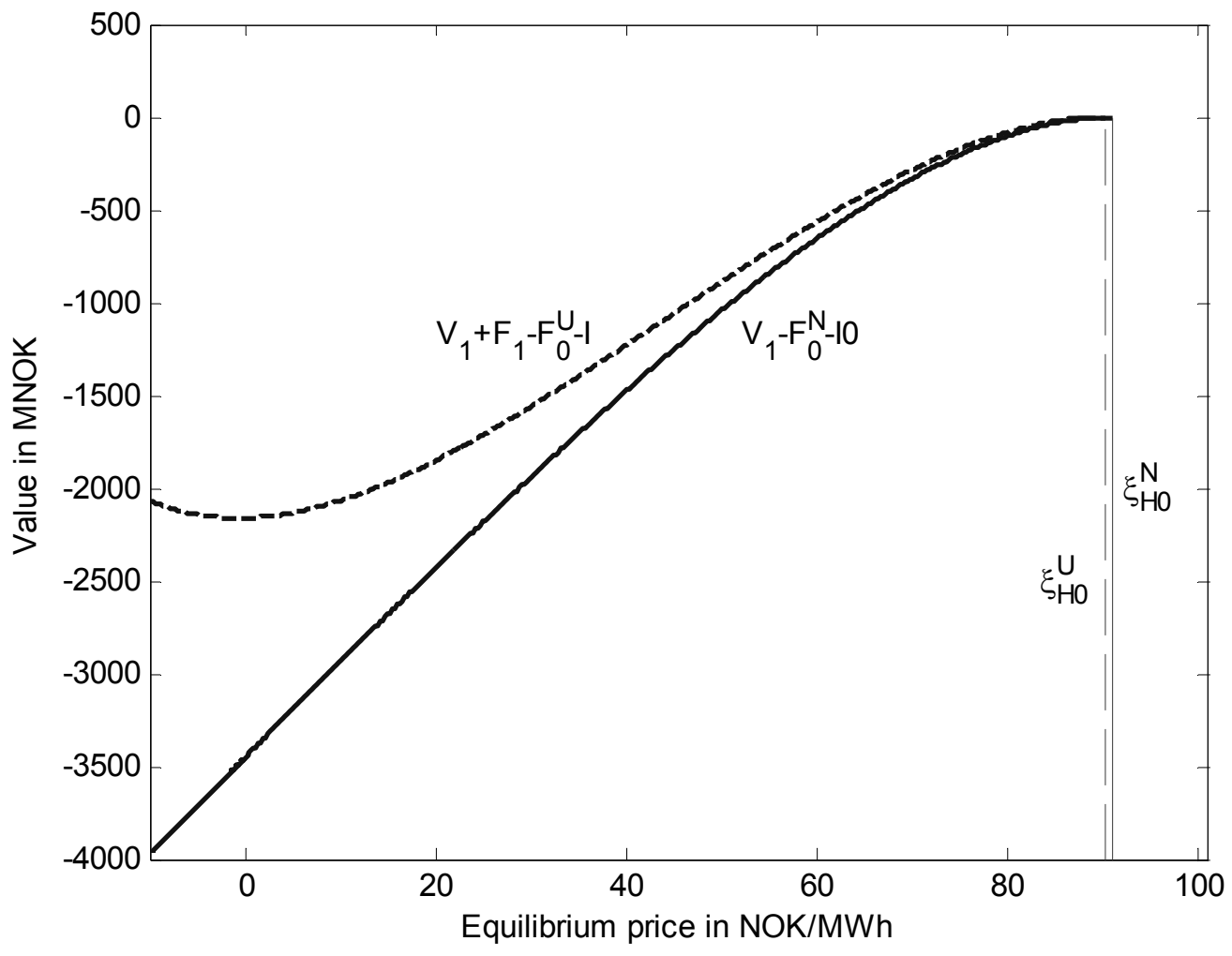

Figure 4

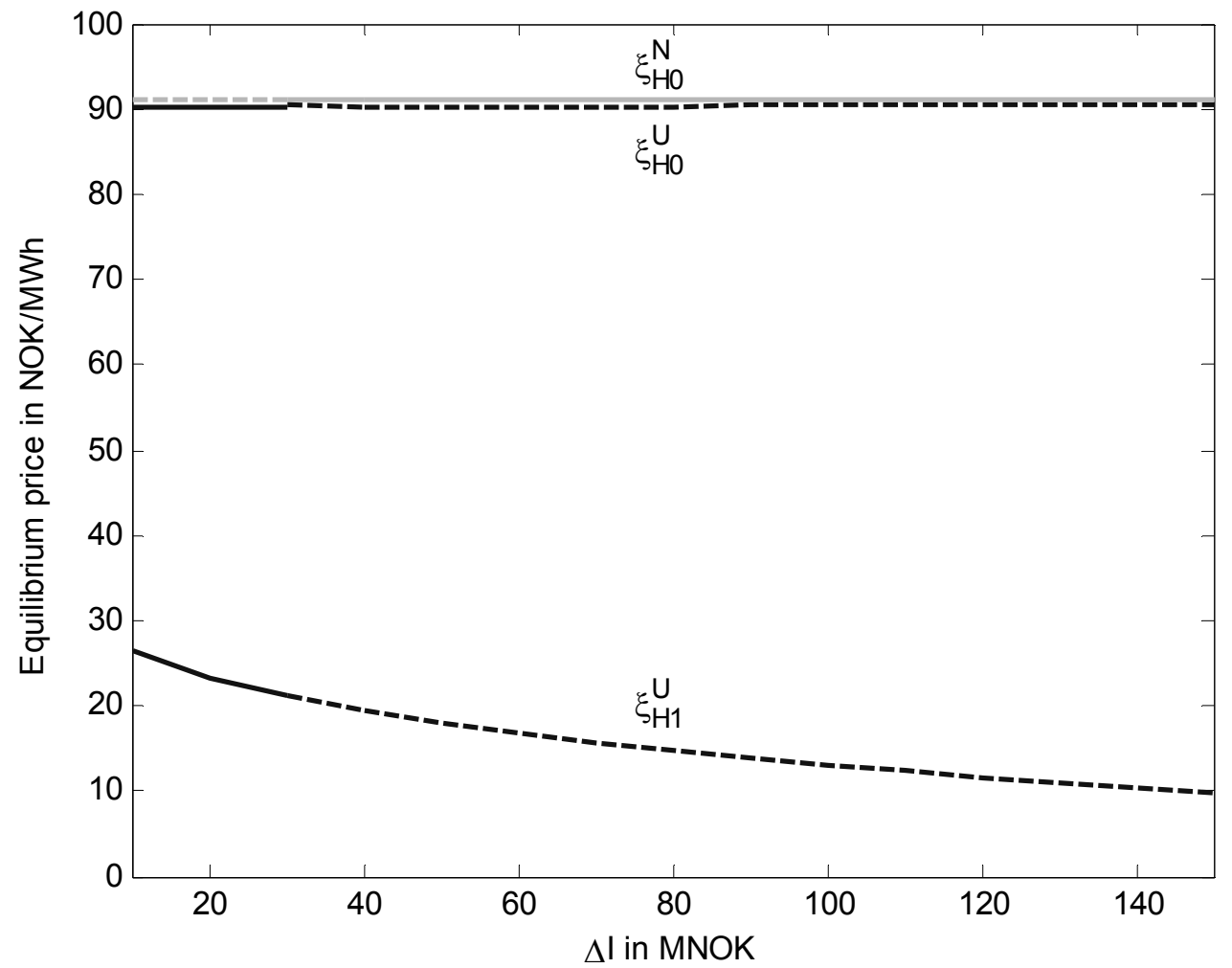

Figure 5 


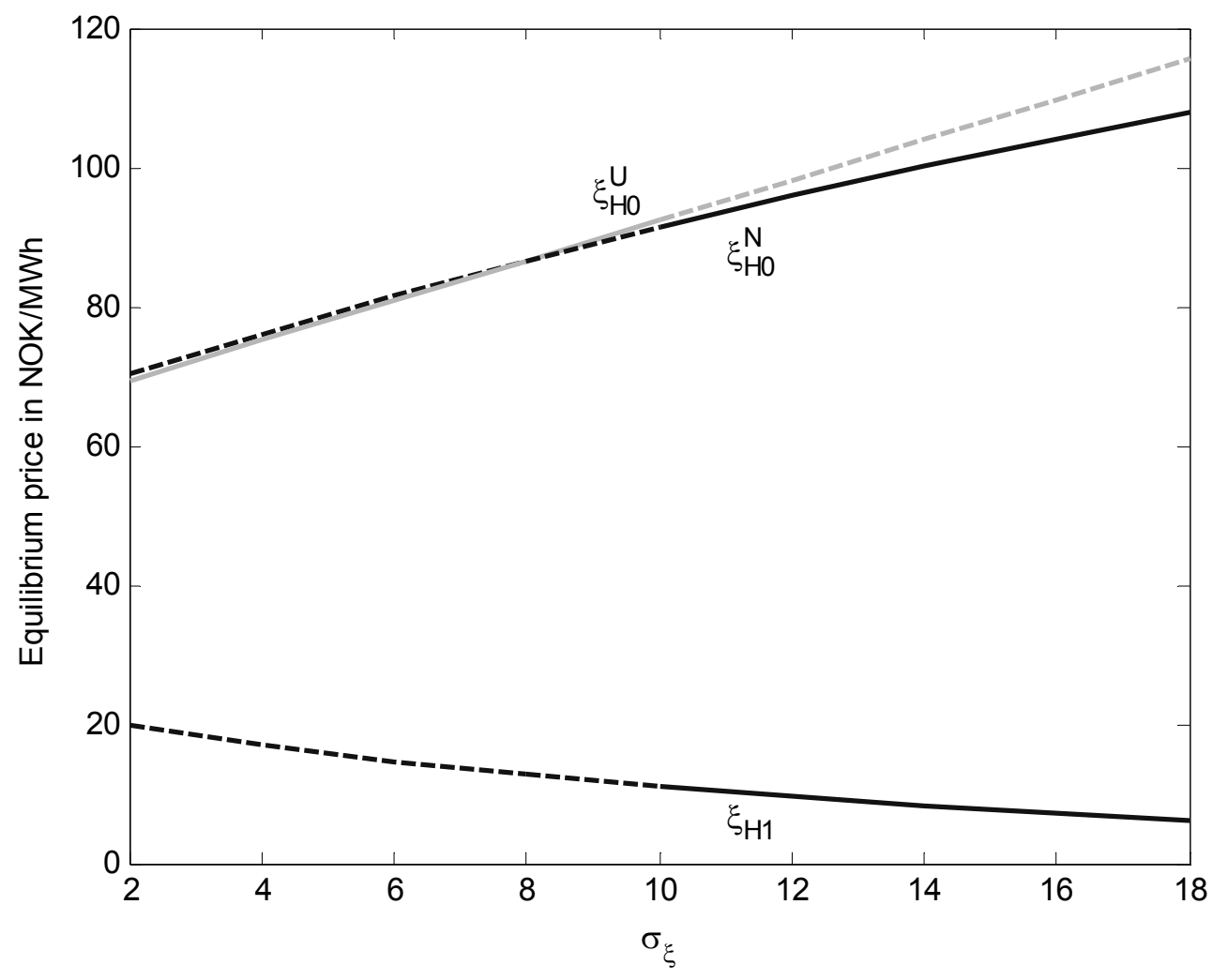

Figure 6

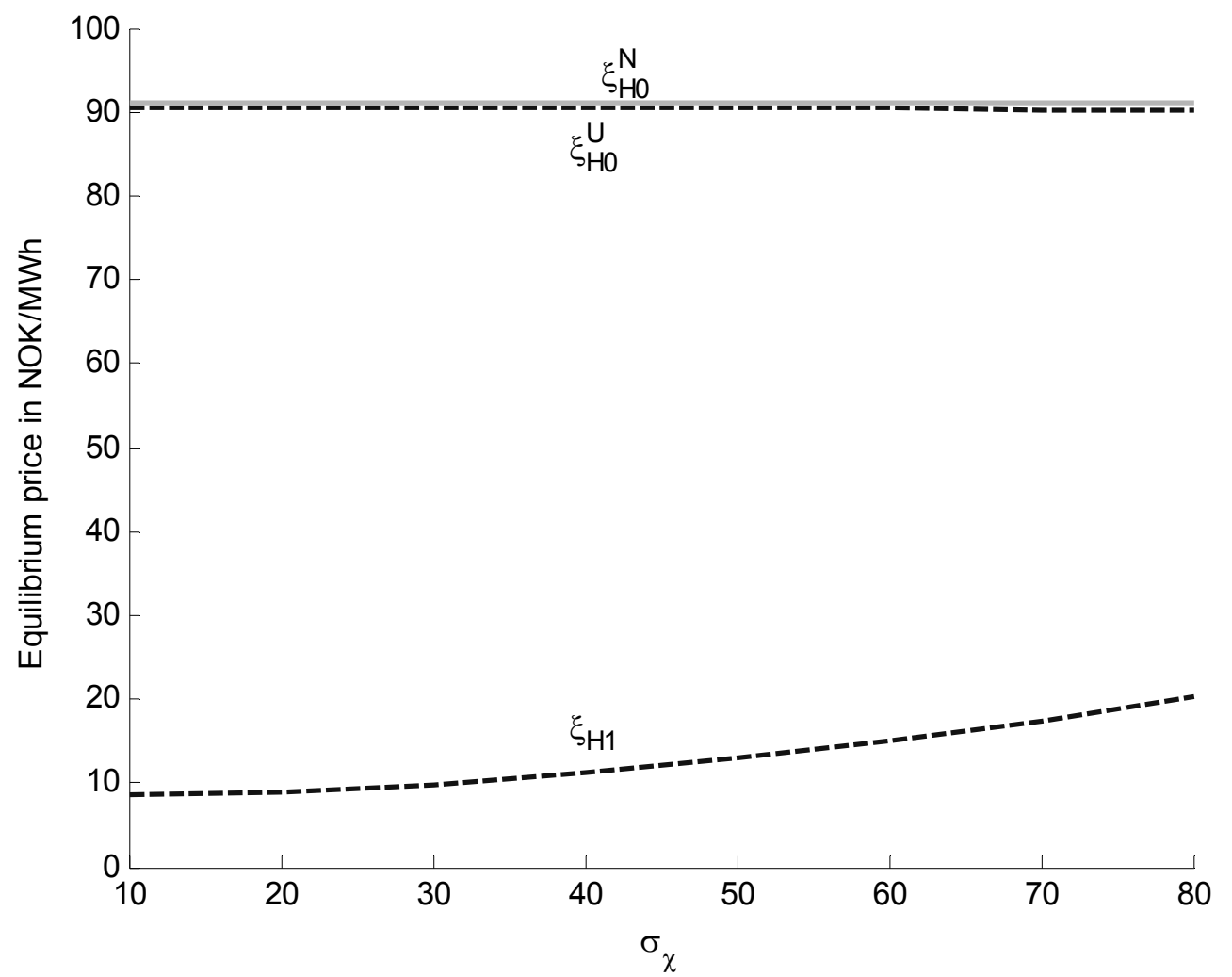

Figure 7 


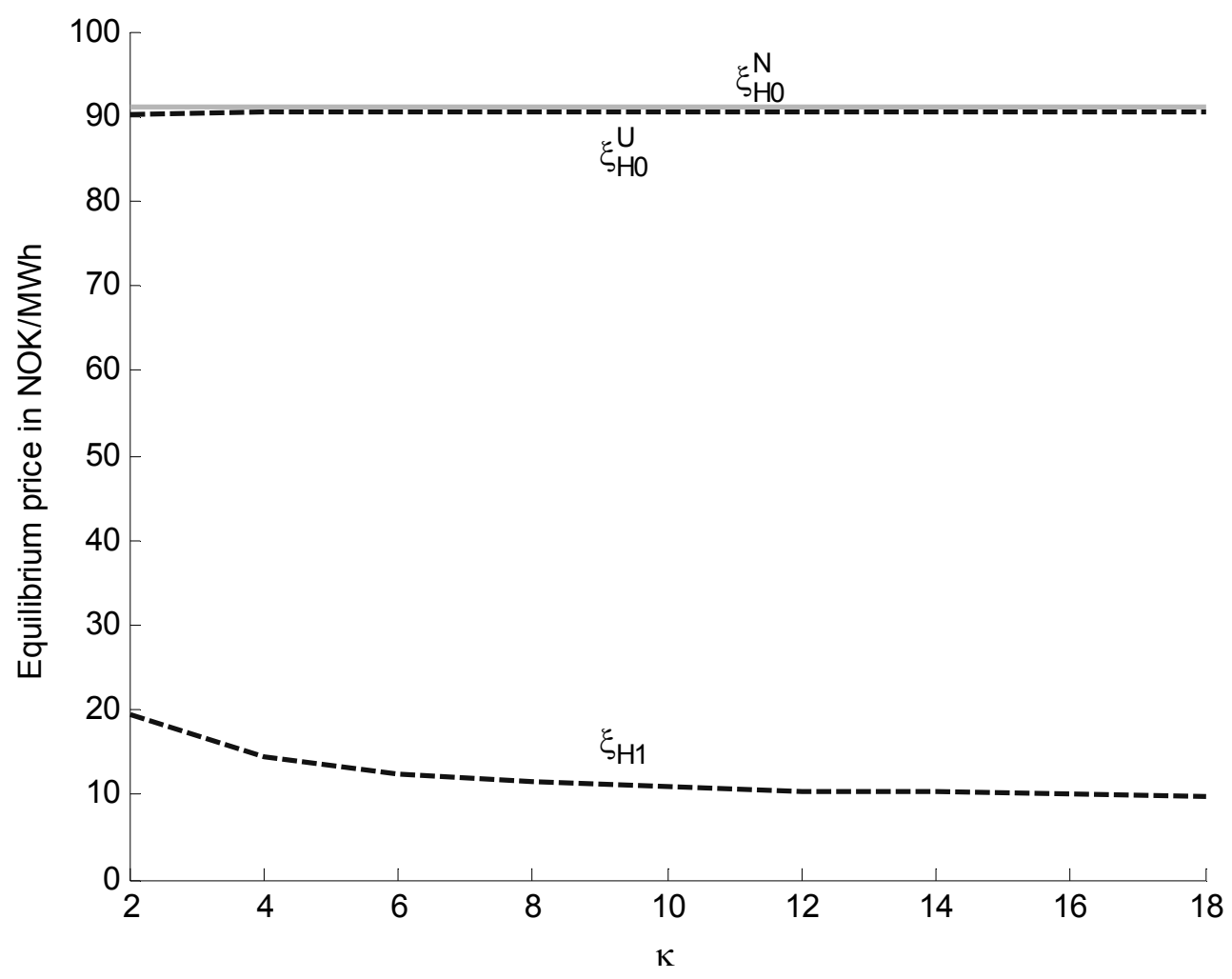

Figure 8 